\title{
Drug persistence and need for dose intensification to adalimumab therapy; the importance of therapeutic drug monitoring in inflammatory bowel diseases
}

Lorant Gonczi ${ }^{1}$, Zsuzsanna Kurti ${ }^{1}$, Mariann Rutka ${ }^{2}$, Zsuzsanna Vegh ${ }^{1}$, Klaudia Farkas ${ }^{2}$, Barbara D. Lovasz ${ }^{1,3}$, Petra A. Golovics ${ }^{1}$, Krisztina B. Gecse ${ }^{1}$, Balazs Szalay ${ }^{4}$, Tamas Molnar ${ }^{2}$ and Peter L. Lakatos ${ }^{1,5^{*}}$

\begin{abstract}
Background: Therapeutic drug monitoring (TDM) aid therapeutic decision making in patients with inflammatory bowel disease (IBD) who lose response to anti-TNF therapy. Our aim was to evaluate the frequency and predictive factors of loss of response (LOR) to adalimumab using TDM in IBD patients.

Methods: One hundred twelve IBD patients (with 214 TDM measurements, CD/UC 84/28, male/female 50/62, mean age CD/UC: $36 / 35$ years) were enrolled in this consecutive cohort from two referral centres in Hungary. Demographic data were comprehensively collected and harmonized monitoring strategy was applied. Previous and current therapy, laboratory data and clinical activity were recorded at the time of TDM. Patients were evaluated either at the time of suspected LOR or during follow-up. TDM measurements were determined by commercial ELISA (LISA TRACKER, Theradiag, France).

Results: Among 112 IBD patients, LOR/drug persistence was 25.9\%/74.1\%. The cumulative ADA positivity (>10 ng/mL) and low TL $(<5.0 \mu \mathrm{g} / \mathrm{mL})$ was $12.1 \%$ and $17.8 \%$ after 1 year and $17.3 \%$ and $29.5 \%$ after 2 years of adalimumab therapy. Dose intensification was needed in $29.5 \%$ of the patients. Female gender and ADA positivity were associated with LOR (female gender: $p<0.001$, OR:7.8 Cl 95\%: 2.5-24.3, ADA positivity: $p=0.007$ OR:3.6 Cl 95\%: 1.4-9.5).

Conclusions: ADA development, low TL and need for dose intensification were frequent during adalimumab therapy and support the selective use of TDM in IBD patients treated with adalimumab. ADA positivity and gender were predictors of LOR.
\end{abstract}

Keywords: Adalimumab, Therapeutic drug monitoring, Inflammatory bowel diseases, Loss of response

\section{Background}

Inflammatory bowel diseases (IBD) are chronic conditions that significantly influence quality of life (QoL) and can lead to disability and complications. Anti-tumor necrosis factor alfa (anti-TNF) therapy is effective in IBD, demonstrating improvement in patients' QoL, leading to clinical remission and mucosal healing, reducing

\footnotetext{
* Correspondence: Peter.Lakatos@muhc.mcgill.ca;

lakatos.peter_laszlo@med.semmelweis-univ.hu; kislakpet99@gmail.com

${ }^{1}$ First Department of Medicine, Semmelweis University, Koranyi S 2A, Budapest H-1083, Hungary

${ }^{5}$ Division of Gastroenterology, McGill University, MUHC, Montreal General Hospital, 1650 Ave. Cedar, D16.173. 1, Montreal, QC H3G 1A4, Canada Full list of author information is available at the end of the article
}

the need for surgery and hospitalizations. However, approximately $10-30 \%$ of the patients do not respond to the initial treatment and app. one third of the patients lose response to anti-TNF therapy over time [1]. There are several possible causes for loss of response (LOR) to anti-TNF therapy, although one of the most common is decreased drug levels due to the development of antidrug antibodies (ADA). ADAs can neutralize the antiTNF drug connecting to the Fab segment of the protein or bind only the anti-TNF molecule promoting the formation of immune complexes both of them leading to increased clearance of the anti-TNF drug through the reticulo-endothelial system. This results in altered drug 
pharmacokinetics and in a reduction of therapeutic efficacy [2]. Therapeutic drug monitoring (TDM), measuring drug trough levels (TL) and ADA levels may aid the therapeutic decision in patients who lose response to anti-TNF therapy. Several studies have indicated that TDM based therapy may predict the loss of response to infliximab therapy [3, 4] and TDM based dosing of infliximab therapy can result in economic benefit [5].

However, less data is available on the relevance of TDM assessment during adalimumab therapy, whether TLs and ADA levels are strongly associated with disease outcome remains questionable. Although correlation between the adalimumab drug concentration and clinical outcome was reported, the role of routine TDM assessment during adalimumab therapy remained unclear [6]. In the post-hoc analysis of the CLinical Assessment of Adalimumab Safety and Efficacy Studied as Induction Therapy in Crohn's Disease (CLASSIC) I/II trial, a positive correlation between serum adalimumab concentrations and clinical remission was reported, although it was not able to delineate a reliable therapeutic cut-off for adalimumab therapy [7]. More recent studies suggested that the optimal therapeutic TL for adalimumab is at around $5 \mu \mathrm{g} / \mathrm{mL}$ [8-10], while different reports exist depending on the measurement methods [2].

The aim of the present study was to evaluate the frequency and predictive factors of loss of response to adalimumab therapy and the role of TDM to predict LOR in adalimumab treated IBD patients.

\section{Methods}

Patients were consecutively enrolled in this cohort between 2014 November and 2016 June from two referral IBD centres in Hungary. Demographic data were comprehensively collected and a harmonized monitoring strategy (including regular assessment of disease activity/biomarkers at start of the adalimumab therapy and during follow-up) was applied as requested by the Hungarian $\mathrm{Na}-$ tional Health Fund [11]. Previous and current therapy, laboratory data and clinical activity at the time of TDM were recorded. Adalimumab was administered at an induction dose of 160/80 $\mathrm{mg}$ and then at standard doses of $40 \mathrm{mg}$ every other week. Dose intensification was defined as administration of $40 \mathrm{mg}$ every week. Samples for TDM were collected right before routine adalimumab injection. Disease location and disease behavior in Crohn's disease (CD) and disease extent in ulcerative colitis (UC) were classified according to the Montreal classification [12].

Patients were evaluated either at the time of suspected LOR (based on clinical evaluation) or during regular follow-up visits with TDM measurement using a conventional and bridging enzyme-linked immunosorbent (ELISA) assay (LISA TRACKER, Theradiag, France). For the measurement of ADA levels, the bridging ELISA method uses a double-antigen bridge: ADAs create a bridge between adalimumab immobilized on the plate and adalimumab enzyme-linked conjugate. ELISA measurements were performed at the Department of Laboratory Medicine, Semmelweis University, Budapest. All ELISA kit was validated for accuracy and reproducibility of TDM for adalimumab (Theradiag, France). The limit of detection for the adalimumab drug level is $0.1 \mu \mathrm{g} / \mathrm{mL}$ determined by the manufacturer of the assay. The optimal therapeutic cut-off value for low adalimumab TL was defined as $5 \mu \mathrm{g} / \mathrm{ml}$, in concordance with previous studies. The standard cut-off value of detectable ADA levels by the assay was $10 \mathrm{ng} / \mathrm{ml}$. To better stratify ADA positive patients we defined ADA levels more than $200 \mathrm{ng} / \mathrm{ml}$ as 'high' ADA level. Transient ADA was defined when the ADA positivity disappeared on the next TDM of the patient. In CD, clinical remission was defined as Crohn's Disease Activity Index (CDAI) $<150$ points or no fistula drainage as assessed by the Fistula Drainage Assessment, while clinical response was defined as a decrease in CDAI with more than 70 points or at least $50 \%$ reduction in the number and production of draining fistulas [13]. In patients with UC, the partial Mayo score (pMayo) was used: patients with more than a 3 point reduction was defined as responder, while patients with pMayo $<3$ were considered as being in remission [14]. LOR was defined as discontinuation of adalimumab therapy. Patients with ongoing symptoms despite dose intensification were discontinued from further adalimumab therapy, and considered to have loss of response. Patients regaining response after dose intensification were considered to have sustained clinical benefit.

\section{Statistical considerations}

For categorical data frequency distributions were analysed, for continuous variables mean and SD were calculated. Chi-squared test was used to evaluate differences within subgroups of patients and binary logistic regression was performed for multivariate analysis (variables with a $p<0.2$ in univariate analysis were included in the multivariate models). For time-dependent outcomes Kaplan-Meier curves were plotted was performed. A $p$-value of $<0.05$ was regarded as statistically significant. Statistical analysis was performed using the SPSS software v. 20.0 (Chicago, IL).

\section{Ethical statement}

The study complies with the principles of the Declaration of Helsinki. The study protocol was approved by the Semmelweis University Regional and Institutional Committee of Science and Research Ethics. (29772-2/2014/EKU).

\section{Results}

Patient characteristics

One hundred twelve IBD patients (with 214 TDM measurements, $\mathrm{CD} / \mathrm{UC} 84 / 28$, male/female $50 / 62$, mean age CD/UC: 36/35 years (y) (SD: 10.9/11.2 y) were enrolled 
in this consecutive cohort. Detailed patient characteristics are shown in Table 1.

The frequency of previous and current 5-aminosalicylate (5-ASA), steroid and azathioprine (AZA) exposure were $85.6 \% / 26.8 \%, 92.9 \% / 6.3 \%$ and $77.7 \% / 44.7 \%$. Previous antiTNF therapy was present in $65.2 \%$ (CD 60.2\%, UC 82.1\%) in the IBD cohort. Mean duration of adalimumab therapy during follow-up was 157.8/70.1 weeks (SD: 101.7/ 58.0 weeks) in $\mathrm{CD} / \mathrm{UC}$.

\section{Frequency of low TL and ADA development}

Among 112 IBD patients, frequency of ADA positivity was $20.5 \%$ (23/112). Among the ADA positive patients, 7 patients had high ADA titres $(>200 \mathrm{ng} / \mathrm{ml})$ and 7 patients had only transient ADA positivity (6.3\%). Frequency of low TL $(<5.0 \mu \mathrm{g} / \mathrm{mL})$ was $31.3 \%$ (35/112). Cumulative ADA positivity was $12.1 \%$ and low TL rate was $17.8 \%$ after 1 year, and $17.3 \%$ and $29.5 \%$, respectively after 2 years of adalimumab therapy. Concomitant immunomodulatory (IM) therapy was not associated with ADA positivity $(p=0.156)$. The combination of normal TL and no ADA, normal TL and ADA positivity, low TL and no ADA, and low TL and ADA positivity were observed in $58 \%, 10.7 \%$, $21.4 \%$ and $9.8 \%$ at TDM measurement (Table 2).

\section{Frequency of dose intensification and loss of response}

Dose intensification was needed in $29.5 \%$ of patients and $25.9 \%$ of the patients had loss of response during the follow-up. The rate of LOR was similar in the two different IBD centres (25.4\% and 26.5\%). In Kaplan-
Meier analysis, probability of dose intensification and LOR was $19.7 \%$ and $17.5 \%$ in the first year and $30 \%$ and $18.8 \%$ in the second year of adalimumab therapy (Figs. 1 and 2).

\section{Predictors for dose intensification, loss of response and TDM levels}

Though the association between low TL and ADA positivity was statistically not significant, the trend towards an association is clear $(47.8 \%$ vs. $27 \%, p=0.054$, OR: 0.4 CI 95\%: 0.2-1.0), furthermore this difference was significant when analysing patients with low and high ADA titre separately. Rate of low TL in patients with high ADA was $85.7 \%$, while it was $28.6 \%$ in patients with low ADA and $27.5 \%$ in ADA negative patients $(p=0.006)$. There was no association between TL and LOR.

ADA positivity was significantly associated with LOR ( $p=0.007$, ADA positivity in LOR $47.8 \%$ vs. $20.2 \%$, OR: 3.6, CI 95\%: $1.4-9.5)$, even if patients with low or high titre ADA (LOR rates in high ADA: 42.9\%, low ADA: $50.0 \%$, no ADA: $20.9 \%, p=0.039$, OR was not available) were analysed separately. The association was significant in CD with a similar trend in UC patients (CD: $52.9 \%$ vs. 23.9\%, $p=0.019$, OR: 3.6 , CI 95\%: $1.2-10.8$, UC: $33.3 \%$ vs. $9 \%, p=0.133$, OR: 5.0 CI 95\%: 0.5-46.7).

Transient ADA was marginally associated with LOR ( $p=0.051$, OR: 4.3, CI 95\%: 0.9-20.4).

LOR was more frequent among patients with dose intensification $(45.5 \%$ vs. $17.7 \%, p=0.002$, OR: 3.869 , CI 95\%: $1.579-9.479)$, especially in $\mathrm{CD}(51.9 \%$ vs. $19.3 \%$,

Table 1 Patient characteristics

\begin{tabular}{|c|c|c|}
\hline & $C D$ & UC \\
\hline Patients number $(n=112)$ & 84 & 28 \\
\hline Males/females & $39 / 45(46 \% / 54 \%)$ & $11 / 17(39 \% / 60 \%)$ \\
\hline Mean age at TDM (SD) & 36 years $(10.9)$ & 35 years (11.2) \\
\hline Mean duration of adalimumab therapy (SD) & 157.8 weeks (101.7) & 70.1 weeks $(58.0)$ \\
\hline Age at onset $(<16 y / 17-40 y />40 y)$ & $13 / 64 / 7$ & - \\
\hline Localisation of Crohn's disease (ileal/colon/ileocolon/upper Gl/ileocolon + upper Gl) & $13 / 16 / 47 / 2 / 5$ & - \\
\hline Extension of colitis (proctitis/left sided/extensive) & - & $0 / 19 / 9$ \\
\hline Behaviour of CD (inflammatory/stricturing/penetrating/strict. + pen.) & 29/18/27/10 & - \\
\hline Perianal disease in CD & $42 / 84(50 \%)$ & - \\
\hline Previous surgery & 43/84 (48.8\%) & $0 / 28(0 \%)$ \\
\hline Smoking (yes/no/previous) & $44 / 24 / 16$ & $19 / 2 / 7$ \\
\hline Previous 5-ASA & 69/84 (82.1\%) & $26 / 28(93 \%)$ \\
\hline Previous steroid & $76 / 84(90.5 \%)$ & $28 / 28(100 \%)$ \\
\hline Previous/concomitant AZA & $67 / 84(80 \%) / 42 / 84(50 \%)$ & $20 / 28(71.4 \%) / 2 / 28(28.6 \%)$ \\
\hline Previous anti-TNF & $50 / 84(60.2 \%)$ & $23 / 28(82 \%)$ \\
\hline Previous IFX/ADM/both & $38 / 5 / 7$ & $22 / 1 / 0$ \\
\hline
\end{tabular}

(CD Crohn's disease, UC Ulcerative colitis, SD Standard deviation, TDM Therapeutic drug monitoring, GI Gastrointestinal, 5-ASA 5-aminosalycilate, AZA Azathioprine, anti-TNF Anti-tumor necrosis factor, IFX Infliximab, ADM Adalimumab) 
Table 2 The combination of ADA and TL status

\begin{tabular}{lll}
\hline & Normal TL & Low TL \\
\hline ADA negative & $58 \%$ & $21.4 \%$ \\
ADA positive & $10.7 \%$ & $9.8 \%$
\end{tabular}

(ADA Anti-drug antibody, TL Trough level)

$p=0.002$, OR: 4.503 CI95\%: 1.655-12.258) with the same trend in UC.

There was a significant association between LOR and female gender $(86.2 \%$ vs. $44.6 \%, p<0.001$, OR: 7.8 CI 95\%: 2.5-24.3) both in CD and UC. In a sensitivity analysis, results remained similar if the data from the two centres were analysed separately. In contrast, there was no association between female gender and dose intensification, low TL or ADA positivity.

Gender ( $p<0.001$, OR: 9.1, 95\% CI: $2.7-30.5)$ and ADA positivity ( $p=0.007$, OR: $4.7,95 \%$ CI: $1.5-14.3)$ remained independent predictors of LOR in a multivariate analysis.

We did not find any association between previous anti-TNF therapy and LOR or ADA status.

Finally, dose intensification was associated with need of steroid therapy in patients with CD $(40.7 \%$ vs. $19.6 \%$, $p=0.04$, OR: 2.8 , CI 95\%: 1.0-7.7).

\section{Discussion}

Results from the present study suggest that ADA development, low TL and need for dose intensification are frequent during adalimumab therapy and support the use of selected TDM assessment in IBD patients during adalimumab therapy.

Need for dose intensification and frequency of LOR was $19.7 \%$ and $17.5 \%$ after 1 year and $30 \%$ and $18.8 \%$ after 2 years of adalimumab therapy. These results are in concordance with the review of Billioud et al. showing that the mean percentage of LOR and dose intensification was $18.2 \%$ and $37 \%$ with an annual risk of $20.3 \%$ and $24.8 \%$ per patient year among primary responders to adalimumab [15]. Nevertheless, in a follow-up study from Leuven, $65.4 \%$ of adalimumab treated patients with previous failure of infliximab therapy needed dose intensification and $38.5 \%$ eventually stopped adalimumab therapy mainly due to LOR [16]. In this study, LOR was more frequent in patients with low TL and high ADA levels during long-term therapy but no predictors for short term clinical response were detected [14].

In the present study, approximately one third of the patients had low TL and $20.5 \%$ had ADA positivity. Patients with high ADA titres $(>200 \mathrm{ng} / \mathrm{mL}$ ) had low TL in $85 \%$. Despite of ADA development, low TL was not

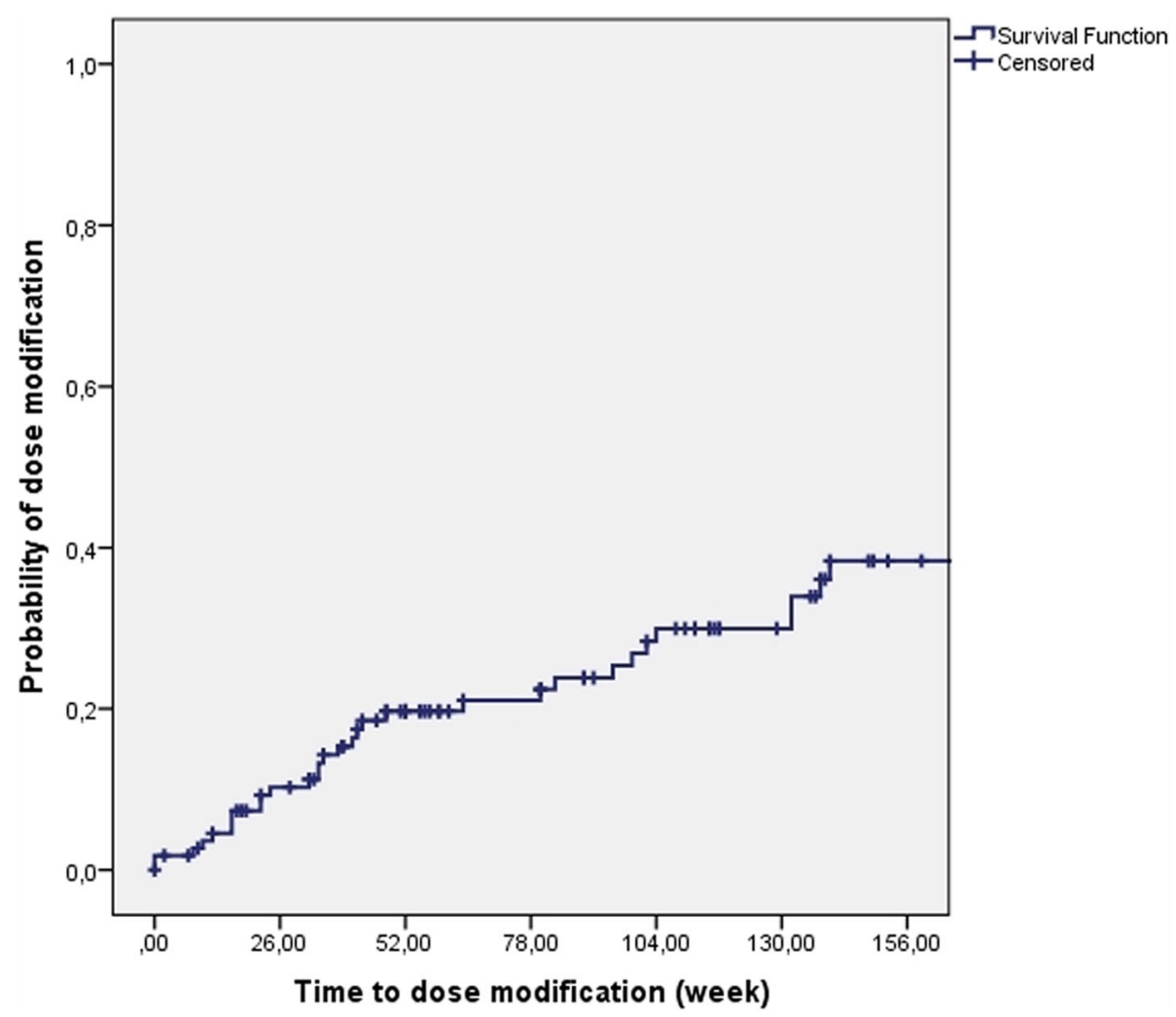

Fig. 1 Probability of dose intensification in Kaplan-Meier analysis 


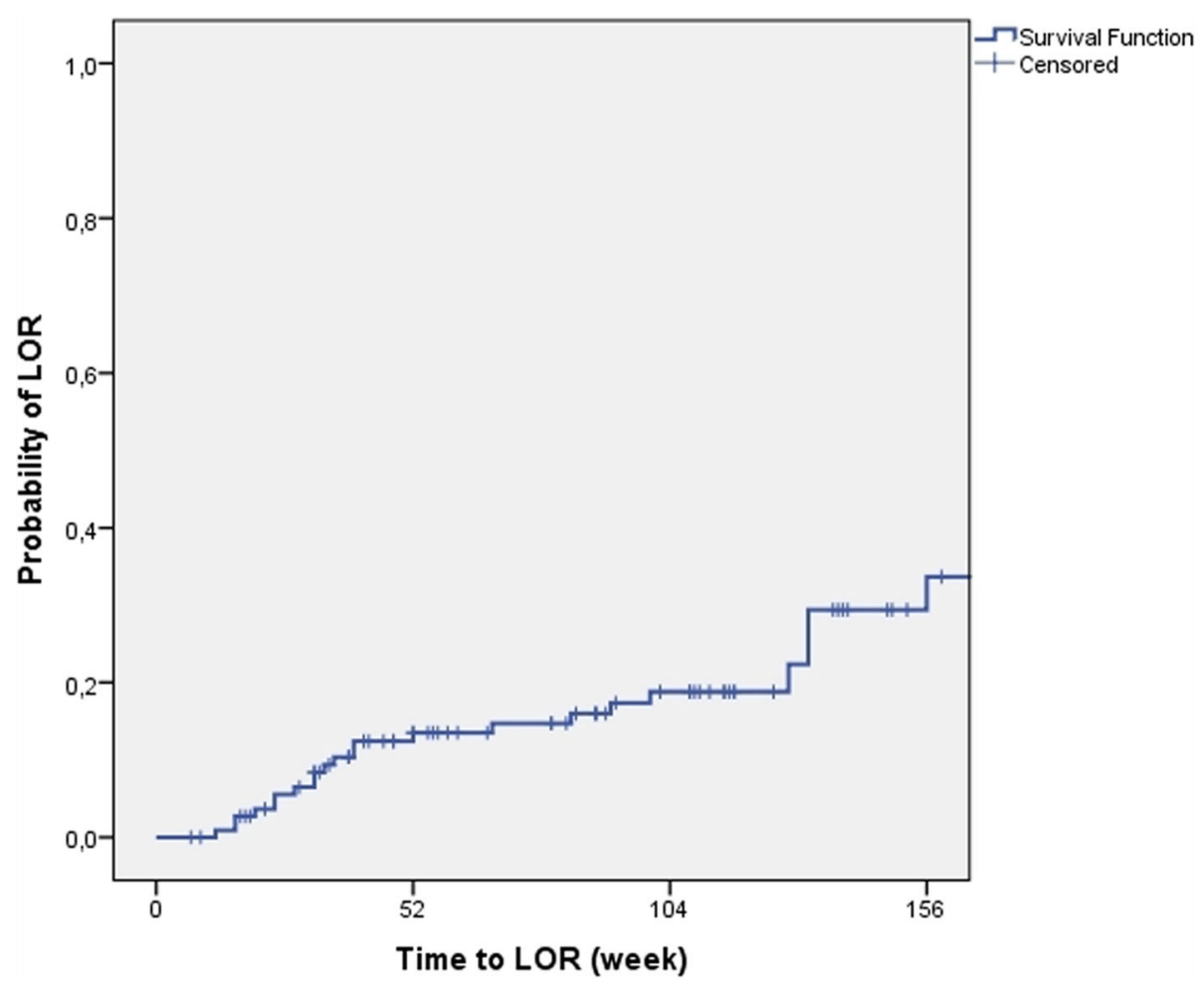

Fig. 2 Probability of LOR in Kaplan-Meier analysis (LOR: loss of response)

associated with LOR. This suggests that primarily ADA positivity should be considered as an indicator for treatment failure. Similarly, Roblin et al. suggested that low TL without ADA can be overcome by dose optimization with a high rate of clinical response (67\%) after dose optimization and is not leading per se to LOR. In contrast, low TL with detectable ADA is associated with treatment failure, and switching to another anti-TNF or other therapeutic class should be considered [17]. Additional articles also suggested that the development of ADA is one of the most important mechanisms of underlying LOR, and routine TDM assessment may help improving patient outcomes. In an Italian study, low TL and ADA positivity correlated with clinical and endoscopic recurrence also in postoperative $C D$ patients [18]. Finally, in a French study, adalimumab TL and duration of treatment were associated independently with mucosal healing, while adalimumab TL, ADA positivity, duration of treatment and adalimumab dose, but not CRP level were associated independently with clinical remission in multivariate analysis [8].

More than half of our patients received another antiTNF agent previously (previous IFX rate: $53 \%$ in CD, $78 \%$ in UC) in the present study. We did not find any association between previous anti-TNF therapy and LOR or ADA status, however the proportion of patients with previous anti-TNF exposure was very high (74/112). In contrast, in the study of Yarur et al., detectable ADA to adalimumab was associated with low adalimumab $\mathrm{TL}$, previous infliximab use, macroscopic mucosal inflammation and need for corticosteroids [19]. Similarly, the positivity rate for ADA to adalimumab was significantly higher in patients who lost response to infliximab (50\%) than in those naive to anti-TNFs (12.5\%) [10].

Concomitant immunomodulatory (IM) therapy was not associated with ADA positivity, in line with previous studies $[9,13,20]$. Few studies reported a benefit of parallel IMs during adalimumab therapy. In a Belgian study, time to dose escalation was longer in patients who were treated with IMs, while Yarur et al. reported that combination therapy of adalimumab and IM yielded a higher mean level of serum adalimumab compared with monotherapy $(14 \mu \mathrm{g} / \mathrm{mL}$ vs $9 \mu \mathrm{g} / \mathrm{mL} ; p=0.026)[14,17]$.

In the present study, there was an association between LOR and female gender both in $\mathrm{CD}$ and $\mathrm{UC}$, even when data from the two referral centres were analysed separately. We did not find gender-related differences in other parameters (e.g. TL, ADA, and severity of disease, pervious and current therapy). In a previous retrospective study by Cohen et al. [21], gender was also associated with dose escalation during adalimumab therapy. Gender related differences were also reported in the rheumatology literature [22, 23]. 
Low adalimumab TL was defined as $<5 \mu \mathrm{g} / \mathrm{mL}$, in concordance with previous studies. However, reported cutoff values vary. Mazor et al. identified a drug TL of $5.85 \mu \mathrm{g} / \mathrm{mL}$ as the optimal cut-off for predicting remission (defined as asymptomatic patients with normal CRP) in CD [9]. In a Japanese study a TL of $5.9 \mu \mathrm{g} / \mathrm{ml}$ was identified to best predict normal CRP in a receiver operator curve (ROC) analysis [10]. Presence of ADA or a serum drug concentration lower than $5 \mu \mathrm{g} / \mathrm{mL}$ was associated with worse self-reported disease symptoms and elevated CRP levels [14, 17]. In contrast, Ungar et al. reported that serum levels of $8-12 \mu \mathrm{g} / \mathrm{mL}$ for adalimumab were required to achieve mucosal healing in patients with IBD. Adalimumab TL higher than $7.1 \mu \mathrm{g} / \mathrm{mL}$ predicted $\mathrm{MH}$ with $85 \%$ specificity in ROC analysis [20].

Current tests for anti-TNF and ADA concentrations are mostly based on enzyme immunoassays. For existing commercially available assays for antibodies, the presence of drug generally interferes with the detection of ADAs. All ADA assays are drug-sensitive to some extent because most assays use the drug itself as labeled detecting antibody. Newer assays, based on high-performance liquid chromatography or the $\mathrm{pH}$ shift-anti-idiotype method, which can detect ADAs in the presence of circulating drug have been developed, however most of these methods only available for infliximab and also not accessible in everyday clinical practice $[24,25]$.

The strengths of the present study are the harmonized monitoring strategy, prospective collection of clinical and laboratory data. Limitations of the study include the relatively small cohort size, especially in UC. Multiple TDM samples were available only in few patients. Of note, unlike infliximab, there are very few studies evaluating serial TDM measurements in adalimumab therapy. Further investigation is needed applying serial/routine TDM measurements to determine the exact role and usability of routine TDM in adalimumab therapy. In addition, whether TDM for adalimumab needs to be performed at trough remains conflictive in current literature and definitely requires more research. Of note, in a very recent paper by Ward et al. author have shown only little variation in drug levels during maintenance adalimumab therapy assessed on days 4-6, 7-9 and 13-14 (trough level). Importantly, drug levels on day 9 were the best predictor of a therapeutic drug trough level [26].

\section{Conclusions}

The present study suggests that ADA development, low $\mathrm{TL}$ and need for dose intensification are frequent during adalimumab therapy and our results support the use of selective TDM assessment in IBD patients on adalimumab therapy. ADA positivity was identified as predictor of loss of response.

\section{Additional file}

Additional file 1: Data set. We provided the dataset of our research as requested by the guidelines of BMC Gastroenterology. All available result of our dataset is presented in the manuscript. (DOCX $90 \mathrm{~kb}$ )

\begin{abstract}
Abbreviations
ADA: Anti-drug antibody; anti-TNF: Anti-tumor necrosis factor alfa; CD: Crohn's disease; CDAl: Crohn's Disease Activity Index; ELISA: Enzyme-linked immunosorbent assay; IBD: Inflammatory bowel disease; LOR: Loss of response; pMayo: Partial Mayo Score; QoL: Quality of life; TDM: Therapeutic drug monitoring; TL: Trough level; UC: Ulcerative colitis
\end{abstract}

\section{Acknowledgements}

Not applicable.

\section{Funding}

This work was supported by OTKA (Hungarian Scientific Research Fund) Research Grant 2015. Grant ID: 115,345.

\section{Availability of data and materials}

All data analysed during this study are included in this published article [and its Additional file 1].

\section{Authors' contributions}

LG and ZK: data collection, data validation, statistical analysis and draft of the article; MR: data collection, data validation; ZV, PAG, BDL, KBG, KF, TM: data collection and article preparation; BS: performed measurements for therapeutic drug level monitoring with enzyme-linked immunoassays; PLL: study design, data collection, supervising the collection and validation of patients, database construction, statistical analysis, and article preparation; All authors approved the final draft submitted.

\section{Ethics approval and consent to participate}

The study complies with the principles of the Declaration of Helsinki. The study protocol was approved by the Semmelweis University Regional and Institutional Committee of Science and Research Ethics (29772-2/2014/EKU). Informed consent to participate in the study were obtained from all participants.

\section{Consent for publication \\ Not applicable.}

\section{Competing interests}

LG, ZK, BS, MR: declare no competing interests. ZV: have been a speaker: AbbVie, Takeda. PAG, BDL: have been a speaker: AbbVie, Ferring, and Takeda. KF: has been speaker for MSD, ABBVIE, and Keri Pharma/Pfizer. TM: has been speaker and advisor for MSD, ABBVIE, Keri Pharma/Pfizer and Takeda. KBG reports having received consultancy fees/speaker activity from Amgen, AbbVie, Ferring, Hospira, MSD, Pfizer, Sandoz, Takeda and Tigenix. PLL has been a speaker and/or advisory board member: AbbVie, EGIS, Falk Pharma GmbH, Ferring, Genetech, Janssen, Kyowa Hakko Kirin Pharma, Mitsubishi Tanabe Pharma Corporation, MSD, Otsuka Pharma, Pharmacosmos, Pfizer, Roche and Takeda and has received unrestricted research grant: AbbVie, MSD and Pfizer; PLL is currently a Section Editor for BMC Gastroenterology. Conference presentation: 11th Congress of ECCO, March 16-19, 2016, Amsterdam, The Netherlands (poster presentation), DDW 2016, May 21-24, 2016, San Diego, California, United States (poster presentation), UEG Week 2016, October 15-19, 2016, Vienna, Austria (poster presentation).

\section{Publisher's Note}

Springer Nature remains neutral with regard to jurisdictional claims in published maps and institutional affiliations.

\section{Author details}

${ }^{1}$ First Department of Medicine, Semmelweis University, Koranyi S 2A, Budapest $\mathrm{H}-1083$, Hungary. ${ }^{2}$ First Department of Medicine, University of Szeged, Szeged, Hungary. ${ }^{3}$ Institute of Applied Health Sciences, Semmelweis University, Budapest, Hungary. ${ }^{4}$ Department of Laboratory Medicine, Semmelweis University, Budapest, Hungary. ${ }^{5}$ Division of Gastroenterology, McGill University, MUHC, Montreal General Hospital, 1650 Ave. Cedar, D16.173. 1, Montreal, QC H3G 1A4, Canada. 
Received: 26 April 2017 Accepted: 31 July 2017

\section{Published online: 08 August 2017}

\section{References}

1. Roda G, Jharap B, Neeraj N, Colombel JF. Loss of response to anti-TNFs: definition, epidemiology, and management. Clin Transl Gastroenterol. 2016;7(1):e135

2. Bodini G, Giannini EG, Furnari M, Marabotto G, Baldissarro I, Del Nero L, et al. Comparison of two different techniques to assess adalimumab trough levels in patients with Crohn's disease. J Gastrointestin Liver Dis. 2015;24(4):451-6.

3. Gonczi L, Vegh Z, Golovics PA, Rutka M, Gecse KB, Bor R, et al. Prediction of short- and medium-term efficacy of biosimilar infliximab therapy. Do trough levels and antidrug antibody levels or clinical and biochemical markers play a more important role? J Crohns Colitis. 2016:12:jiw203. [Epub ahead of print].

4. Kobayashi T, Suzuki Y, Motoya S, Hirai F, Ogata H, Ito H, et al. First trough level of infliximab at week 2 predicts future outcomes of induction therapy in ulcerative colitis - results from a multicenter prospective randomized controlled trial and its post hoc analysis. J Gastroenterol. 2016;51(3):241-51.

5. Vande Casteele N, Ferrante M, Van Assche G, Ballet V, Compernolle G, Van Steen $\mathrm{K}$, et al. Trough concentrations of infliximab guide dosing for patients with inflammatory bowel disease. Gastroenterology. 2015;148(7):1320-9.e3.

6. Casteele NV, Feagan BG, Gils A, Vermeire S, Khanna R, Sandborn WJ, et al. Therapeutic drug monitoring in inflammatory bowel disease: current state and future perspectives. Curr Gastroenterol Rep. 2014;16(4):378.

7. Chiu YL, Rubin DT, Vermeire S, Louis E, Robinson AM, Lomax KG, et al. Serum adalimumab concentration and clinical remission in patients with Crohn's disease. Inflamm Bowel Dis. 2013;19(6):1112-22.

8. Roblin X, Marotte H, Rinaudo M, Del Tedesco E, Moreau A, Phelip JM, et al. Association between pharmacokinetics of adalimumab and mucosal healing in patients with inflammatory bowel diseases. Clin Gastroenterol Hepatol. 2014;1:80-4

9. Mazor Y, Almog R, Kopylov U, Ben Hur D, Blatt A, Dahan A, et al. Adalimumab drug and antibody levels as predictors of clinical and laboratory response in patients with Crohn's disease. Aliment Pharmacol Ther. 2014:40(6):620-8.

10. Imaeda H, Takahashi K, Fujimoto T, Bamba S, Tsujikawa T, Sasaki M, et al. Clinical utility of newly developed immunoassays for serum concentrations of adalimumab and anti-adalimumab antibodies in patients with Crohn's disease. J Gastroenterol. 2014;49:100-9.

11. Gecse KB, Lovasz BD, Farkas K, Banai J, Bene L, Gasztonyi B, et al. Efficacy and safety of the biosimilar infliximab CT-P13 treatment in inflammatory bowel diseases: a prospective, multicentre, nationwide cohort. J Crohns Colitis. 2016;10(2):133-40

12. Silverberg MS, Satsangi J, Ahmad T, Arnott ID, Bernstein CN, Brant SR, et al. Toward an integrated clinical, molecular and serological classification of inflammatory bowel disease: report of a working party of the 2005 Montreal world congress of gastroenterology. Can J Gastroenterol. 2005 Sep;19(Suppl A):5A-36A.

13. Best WR, Becktel JM, Singleton JW. Rederived values of the eight coefficients of the Crohn's disease activity index [CDAI]. Gastroenterology. 1979;77:843-6

14. Rutgeerts P, Sandborn WJ, Feagan BG, et al. Infliximab for induction and maintenance therapy for ulcerative colitis. N Engl J Med. 2005;353:2462-76.

15. Billioud V, Sandborn WJ, Peyrin-Biroulet L. Loss of response and need for adalimumab dose intensification in Crohn's disease: a systematic review. Am J Gastroenterol. 2011;106(4):674-84.

16. Karmiris K, Paintaud G, Noman M, Magdelaine-Beuzelin C, Ferrante M, Degenne D, et al. Influence of trough serum levels and immunogenicity on long-term outcome of adalimumab therapy in Crohn's disease. Gastroenterology. 2009;137:1628-40

17. Roblin X, Rinaudo M, Tedesco E, Phelip JM, Genin C, Peyrin-Biroulet L, et al. Development of an algorithm incorporating pharmacokinetics of adalimumab in inflammatory bowel diseases. Am J Gastroenterol. 2014;109:1250-6.

18. Bodini G, Savarino V, Peyrin-Biroulet L, de Cassan C, Dulbecco P, Baldissarro I, et al. Low serum trough levels are associated with post-surgical recurrence in Crohn's disease patients undergoing prophylaxis with adalimumab. Dig Liver Dis. 2014;46:1043-6.
19. Yarur AJ, Deshpande AR, Sussman DA, Hauenstein S, Lockton S, Barkin JS, et al. Serum adalimumab levels and antibodies correlate with endoscopic intestinal inflammation and inflammatory markers in patients with inflammatory bowel disease. Gastroenterol Hepatol (N Y). 2013:9(8, Suppl 4):1-16.

20. Ungar B, Levy I, Yavne Y, Yavzori M, Picard O, Fudim E, et al. Optimizing anti-TNF-a therapy: serum levels of infliximab and adalimumab are associated with mucosal healing in patients with inflammatory bowel diseases. Clin Gastroenterol Hepatol. 2016;14(4):550-7.

21. Cohen RD, Lewis JR, Turner H, Hanauer SB, Rubin DT. Predictors and timing of adalimumab (ADA) dose escalation in patients with Crohn's disease (CD). Gastroenterology. 2009;136:A-652.

22. Jawaheer D, Maranian P, Park G, Lahiff M, Amjadi SS, Paulus HE. Disease progression and treatment responses in a prospective DMARD naive seropositive early rheumatoid arthritis cohort: does gender matter? J Rheumatol. 2010;37(12):2475-85.

23. Gremese E, Bernardi S, Bonazza S, Nowik M, Peluso G, Massara A, et al. Body weight, gender and response to TNF-alpha blockers in axial spondyloarthritis. Rheumatology (Oxford). 2014;53(5):875-81.

24. Wang S, Ohrmund L, Ling N, et al. Analysis of anti-drug antibodies (ADA) to adalimumab in patient serum using a novel homogeneous mobility shift assay. Am J Gastroenterol. 2010;105(suppl 1):S444-5.

25. Schouwenburg V. Van Schouwenburg P, Bartelds G, Hart M, et al. a novel method for the detection of antibodies to adalimumab in the presence of drug reveals "hidden" immunogenicity in rheumatoid arthritis. J Immunol Methods. 2010;362:82-8.

26. Ward MG, Thwaites PA, Beswick L, Hogg J, Rosella G, Van Langenberg D, Reynolds J, Gibson PR, Sparrow MP. Intra-patient variability in adalimumab drug levels within and between cycles in Crohn's disease. Aliment Pharmacol Ther. 2017:45:1135-14.

\section{Submit your next manuscript to BioMed Central and we will help you at every step:}

- We accept pre-submission inquiries

- Our selector tool helps you to find the most relevant journal

- We provide round the clock customer support

- Convenient online submission

- Thorough peer review

- Inclusion in PubMed and all major indexing services

- Maximum visibility for your research

Submit your manuscript at www.biomedcentral.com/submit
Biomed Central 